\title{
Guest editorial: Fetal growth restriction and its consequences
}

\author{
Vandana Jain • Vinod Kumar Paul
}

Published online: 25 April 2012

(C) Springer Science+Business Media, LLC 2012

Worldwide, more than 30 million infants are born with low birth weight each year. India contributes the lion's share with more than 8 million low weight births annually. A majority of these are born at term but have experienced fetal growth restriction. While a proportion of these cases can be ascribed to recognizable etiological mechanisms, such as maternal malnutrition, systemic illness or pregnancy related complications, no definite cause can be ascertained in a vast majority. These infants are at increased risk of mortality and morbidity in early life; as well as a greater later risk of developing central obesity, dyslipidemia, hypertension, insulin resistance and diabetes, as well as short stature and early puberty.

Over the past many years, research efforts to elucidate the biology of normal and abnormal growth have not been commensurate with the magnitude of the problem. There has been a paucity of novel/path-breaking research for identification of underlying mechanistic pathways as well as successful interventions for prevention of fetal growth restriction and its adverse consequences. However, with recent technological advances in the understanding of genetic/ epigenetic pathways and availability of sophisticated bioinvestigation tools, there is an opportunity to make renewed research efforts in this direction.

\section{Jain}

Division of Pediatric Endocrinology, Department of Pediatrics, All India Institute of Medical Sciences,

New Delhi, India

e-mail: drvandanajain@gmail.com

\section{K. Paul ( $\varangle)$}

Department of Pediatrics and WHO Collaborating Centre for Training and Research in Newborn Care,

All India Institute of Medical Sciences,

New Delhi, India

e-mail: vinodkpaul@hotmail.com
An international scientific meeting titled "Consultation on Biology of Fetal Growth Restriction and its Consequences" was organized at the All India Institute of Medical Sciences, New Delhi from 23rd to 25th November, 2010, with the aim to review the current status of research, and to define and prioritize research ideas in this field. The meeting saw active participation by experienced researchers, policy-makers and funding agencies, including National Institute of Health, USA, Indo-US Science and Technology Forum, and the Departments of Biotechnology and Health Research of Government of India.

The deliberations of this meeting included wide-ranging discussions on themes such as role of endocrine pathways and one carbon metabolism in regulation of fetal growth, effect of maternal micronutrient deficiency/supplementation on growth and metabolism in the offspring, the 'omics' approach to explore pathways of fetal growth restriction, developmental programming of body composition, and the potential mechanisms underlying developmental origins of diseases.

The most important recommendations that emerged from the meeting were that there is an urgent need to conduct cohort studies to a), identify fetal growth patterns and determinants of fetal growth restriction in different populations and b), to prospectively study early growth trajectories of small for gestational age infants in resource-limited settings to identify growth patterns that optimize the risk to benefit balance in terms of stature, body composition, risk of infections, undernutrition, later cardiovascular/diabetes risk and neuro-cognitive development. It was felt that India, with its high burden of fetal growth restriction, a population in transition (economic, nutritional and socio-demographic), tremendous science capacity as well as enhanced budgetary allocation for health research by the Government, is uniquely poised to undertake significant research in this field. 
This special issue of REMD consists of seven papers contributed by the eminent faculty for this meeting. Kalhan and Marczewski have described the impact of perturbations in methionine and homocysteine metabolism in the mother on fetal growth. Yajnik and Deshmukh have presented the findings of their longitudinal study in Pune, India, that showed that maternal vitamin B12 deficiency is associated with small size at birth, and greater adiposity and insulin resistance at the age of 6 years. Rao, Padmavathi and Raghunath have reviewed the role of maternal micronutrient deficiencies in the fetal origins of adiposity and insulin resistance, based on animal research models. Jain and Singhal have addressed the controversy regarding the risk versus benefit of catch-up growth in low birth weight infants, especially from an Indian perspective, where a high prevalence of infectious illnesses and undernutrition in childhood co-exists with very high rates of type 2 diabetes and cardiovascular disease in adults. Wells has discussed the value of assessing body composition in infants for resolving the uncertainty regarding the effect of infancy nutrition on later health; and also comprehensively reviewed the available techniques. Martin-Gronert and Ozanne have discussed some of the biological mechanisms that underlie developmental programming of health and disease. Lastly, Roth and Sathyanarayana have reviewed the neuroendocrine pathways and epigenetic modifications that may have potentially important roles in the altered regulation of body weight and pubertal onset in children born small for gestational age.

We wish to express our sincere gratitude to all the authors who have contributed to this special issue. We would also like to thank the editors of 'Reviews in Endocrine and Metabolic Disorders' for providing us with this opportunity to put together some of the recent insights in the field of fetal growth restriction and its consequences. 